\section{Lateral hypothalamic stimulation: Experience and deprivation as factors in rat's licking of empty drinking tubes}

\author{
DENNISON A. SMITH* \\ University of Massachusetts, Amherst, Massachusetts 01002
}

Animals' behavior when 24 or $48 \mathrm{~h}$ food deprived was compared with their behavior when given high or low electrical stimulation of the lateral hypothalamus to see if the previous experience of drinking sucrose from tubes could induce the animals in both conditions to respond on the tubes when empty. Only in the electrical stimulation condition did the Ss show any significant responding on the empty tubes.

Valenstein, Cox, \& Kakolewski (1969) have presented data which suggest that motivational states elicited by electrical stimulation of the lateral hypothalamus (ESLH) do not resemble those that result from deprivation. Valenstein et al (1969) have suggested that ESLH creates "a condition which increases the incidence of well-established motor responses." In one study that suggests this conclusion, Valenstein et al showed that rats stimulated in the lateral hypothalamus will persist in licking an empty drinking tube from which they drank during previous periods of lateral hypothalamic stimulation. Presumably, rats not given electrical stimulation of the lateral hypothalamus, whether deprived or nondeprived, do not exhibit such behavior. However, nowhere do they discuss the behavior of a nonstimulated control group as a basis for comparison. Furthermore, all of the animals tested by these Es had had extensive experience drinking from tubes when given ESLH. It is possible that the persistent drinking behavior of the brain-stimulated animals on the empty drinking tubes is also shown by the animals when not motivated by ESLH. Similarly, the -extensive experience of drinking from tubes might lead to the licking of the tubes when they are empty. For example, Moll (1969) has shown that with repeated experience in a test situation, rats increase their intake of food. Le Magnen (1967) has pointed out that animals easily develop position habits toward food and also develop food acquisition habits related to the environment from which they obtained food.

This experiment makes a direct test of these ideas. Specifically, rats that have had extensive experience licking sucrose from drinking tubes during

*Now at Oberlin College. Requests for reprints should be sent to Dennison Smith, Department of Psychology. Oberlin College, Oberlin, Ohio 44074 . periods with and without brain stimulation will be tested to see if this switch from the original response of tube licking to a new response when the drinking tubes are empty.

\section{SUBJECTS}

Eight Charles River albino rats were selected from a larger group of rats $(\mathrm{N}=30)$ that were stereotaxically implanted with electrodes aimed at the perifornical hypothalamus. The eight Ss selected showed either stimulus-bound eating, gnawing, or drinking during initial screening trials experiment, Ss were individually housed under constant lighting conditions and were maintained on a regimen of ad lib Purina Lab Chow and water, except on those days designated by the experimental design. SURGERY-HISTOLOGY

The Ss were anesthetized with Nembutal $(40 \mathrm{mg} / \mathrm{kg})$ and implanted bilaterally with stainless steel monopolar electrodes insulated except at their tips. The electrodes were aimed at an area dorsolateral to the hypothalamic fornix. Krieg (1946) stereotaxic coordinates were used. They were: $.8 \mathrm{~mm}$ posterior to bregma, $1.6 \mathrm{~mm}$ to the left and right of midline, and $8.5 \mathrm{~mm}$ vertical to brain top. A jeweler's screw attached to the skull served as the ground.

On completion of the experiment, the Ss were sacrificed with an overdose of Nembutal anesthesia and perfused with physiological saline and $10 \%$ formalin. The brains were then frozen, and 30 -micron sections were cut through the extent of the electrode tract. Every fifth section was stained with cresyl violet and mounted on a slide.

\section{APPARATUS}

The rats were tested in a $12 \times 12 \times 1218$ in.-high box, placed in a closet specially prepared for sound attenuation.

Drinkometer cireuits (Grason-Stadler Model Nos. E4690A-1 experience influences their ability to with ESLH. Throughout the and E4690A-2) were used to record licking responses on two drinking tubes attached side by side to one side of the box. The electrical brain stimulation was provided by a step-down transformer operated from a $110-\mathrm{V}$ ac line. The current was regulated by an ac potentiometer in series with the rat and monitored on an ac microammeter. PROCEDURE

Screening and Licking Training

After a 7-day postoperative recovery period, all animals were screened. The screening procedure consisted of stimulating the rats in the experimental chamber in the presence of Purina rat pellets, a wood block, and a drinking tube filled with tap water. The stimulation current was increased from zero in 5-microA steps until stimulus-bound eating, drinking, or gnawing was observed or until the stimulation became aversive. The stimulus was judged to be aversive when Ss attempted to escape by jumping or when seizures occurred. During this procedure, the current was turned on manually, but it was never left on for longer than $1 \mathrm{~min}$.

Immediately following screening, all the rats that showed a stimulus-bound behavior were tested to see if their behavior could be switched to stimulus-bound drinking of a $30 \%$ sucrose solution. With only sucrose available in the drinking tubes, the animals were subjected to 60-sec periods of ESLH at stimulus intensities at which they had first showed stimulus-bound behavior. A 30-sec intertrial interval (ITI) was employed. All Ss switched their behavior to stimulus-bound drinking within $1 \mathrm{~h}$ or less. High and low levels of brain stimulation were selected for each animal. The criteria for selecting the intensities were: (1) that the low intensity was the lowest that yielded stimulus-bound drinking on every trial and (2) that the high intensity was the highest that yielded consistent drinking and that did not produce agitation that significantly interfered with the drinking. All Ss were then given an additional $2 \mathrm{~h}$ of training. During one of the hours the high intensity stimulation was used, and during the other hour low intensity stimulation was used.

Additional drinking training in the test chamber followed. Every third day for a 12-day period (4 training days), the Ss were assigned randomly to one of four training conditions. During each training day, each rat experienced a situation in which drinking could occur without brain stimulation and a situation in which drinking was elicited by ESLH. Each training segment consisted of $12 \mathrm{~min}$ of drinking tube availability. In the 

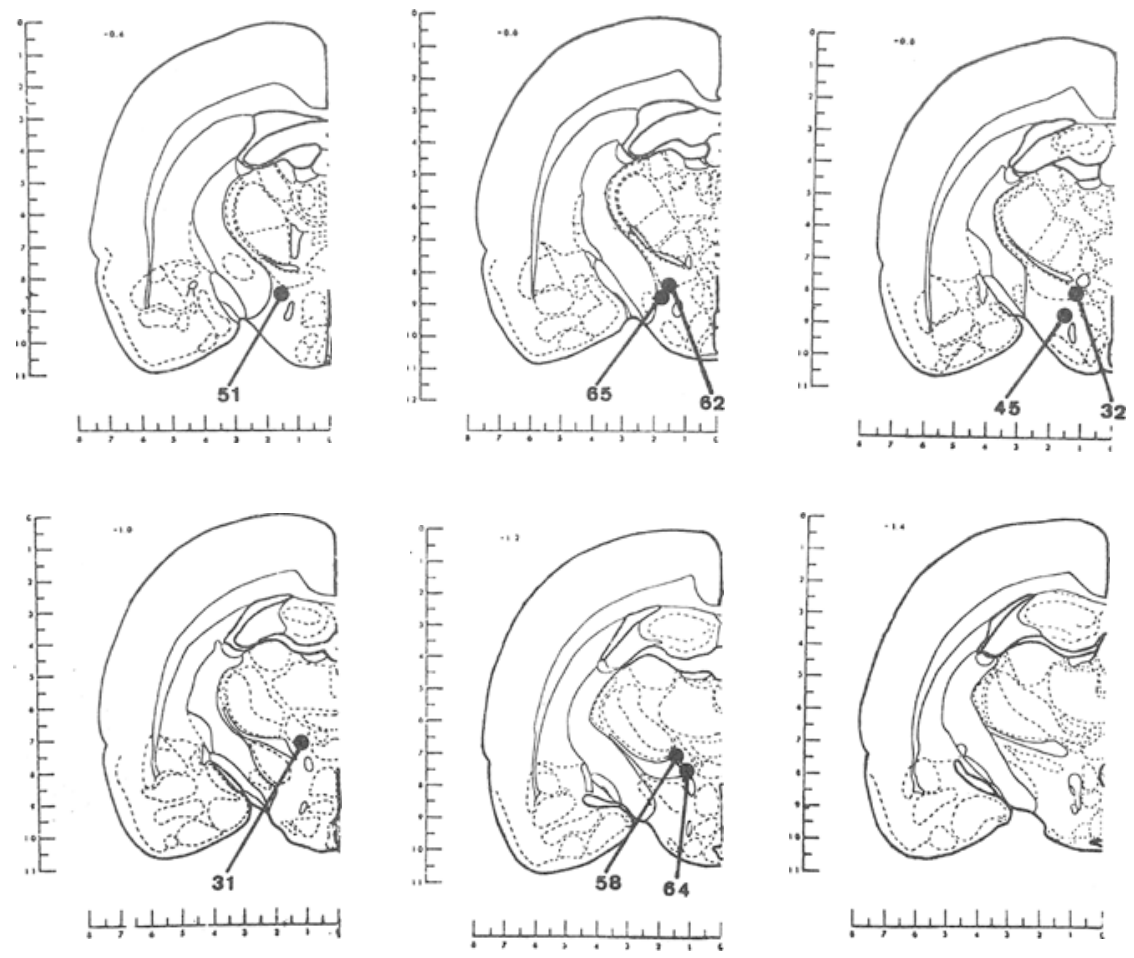

Fig. 1. Localization of electrode tips.

ESLH segment, brain stimulation was turned on for $2 \mathrm{~min}$ and turned off for $30 \mathrm{sec}$ before coming on again for the next 2 min (six trials).

The following conditions were e mployed: (1) 24-h food deprivation/high ESLH, (2) 24-h deprivation/low ESLH, (3) 48-h deprivation/high ESLH, and (4) 48-h deprivation/low ESLH. Tests under deprivation conditions were always given first, and at least $4 \mathrm{~h}$ passed, during which time the rats were on ad lib food and water, before the animals were given training with ESLH.

\section{Testing}

The behavior of each of the Ss was investigated on two separate test days spaced 2 days apart to allow for the manipulation of the deprivation conditions. On one of the days, the rat's behavior was examined during a 5-min period of continuous brain stimulation. Rats were placed randomly in either the high- or low-ESLH groups (four per group). On the rat's other test day, his behavior was recorded for $5 \mathrm{~min}$ while he was either 48- or 24-h deprived. One $S$ was assigned to each of eight possible test sequences.

Within the test chamber, a dish with $200 \mathrm{~g}$ of wet mash was situated between the two, now empty, drinking tubes. At the beginning of each test session, a metal barrier that separated the rat from the drinking tubes and wet mash was removed. On ESLH trials, stimulation also began at this time. Minute by minute records of the licks on the empty drinking tubes were recorded.

\section{RESULTS}

Figure 1 presents a summary of the brain areas from which stimulus-bound behavior was elicited. For the most part, electrodes were located dorsolateral to the fornix and mammillothalamic tract within the lateral hypothalamus or zona incerta.

Even casual examination of Table 1 demonstrates that neither deprivation nor the previous experience of licking in this situation produced responding on the empty tubes. However, in the ESLH condition the rats persisted in responding on the empty tubes despite the fact that food was readily available in the foodcup. Sign tests (Siegel, 1956) demonstrate significant differences between brain stimulation and both deprivation conditions ( $p<.01)$. Furthermore, it is evident that the licking on the empty tubes drops off significantly over the 5-min period of electrical stimulation. For instance, a comparison between the number of licks during the first minute with the number of licks in the fifth minute shows significant differences (sign test, $p<.01$ ). There is a tendency for higher intensity stimulation to evoke more licking behavior on the empty tubes. However, there is a great deal of variability, especially in the low brain-stimulation group, which prevents any conclusion on this point. No significant differences were observed when comparing the two deprivation conditions.

Measures of the latency to the first bite of food and the number of grams of wet mash consumed proved unreliable. Typically, the ESLH condition produced quite variable results. Often, short latencies for eating wet mash were observed. However, the mash was quickly abandoned, and at least for the first $2 \mathrm{~min}$, the rats showed comparatively more behavior for the empty drinking tubes. Under ESLH conditions, a good deal of searching and other extraneous behavior accompanied the discovery of the empty drinking tubes. The Ss often rapidly shifted their responding from one tube to the other. After several minutes of ESLH, most of the ESLH Ss eventually began to consume the wet mash consistently.

With ESLH, the animals' initial eating responses of wet mash were quite diffuse and not well integrated. After some experience, the animals appeared to be less active and the food was consumed less ravenously. The amounts of food consumed by the brain-stimulated rats also proved to be unreliable because a good deal was scattered as the animals ate. This pattern of behavior contrasts sharply with the behavior of the same animals when deprived. When deprived, the animals rarely paid any attention to anything but the wet mash, which they began to eat almost immediately.

Table 1

Licking Responses on Empty Tubes

\begin{tabular}{|c|c|c|c|c|c|}
\hline \multirow{2}{*}{$\begin{array}{c}\text { Rat } \\
\text { Number }\end{array}$} & \multicolumn{5}{|c|}{ Minutes } \\
\hline & 1 & 2 & 3 & 4 & $\mathbf{5}$ \\
\hline \multicolumn{6}{|c|}{ High Brain Stimulation } \\
\hline 32 & 31 & 52 & 32 & 37 & $\mathbf{0}$ \\
\hline 51 & 67 & 35 & 28 & $\mathbf{0}$ & 6 \\
\hline 62 & 35 & 26 & 8 & 11 & 1 \\
\hline 65 & 32 & 26 & 2 & 3 & $\mathbf{0}$ \\
\hline Total & 165 & 139 & 70 & 51 & 7 \\
\hline \multicolumn{6}{|c|}{ Low Brain Stimulation } \\
\hline 31 & 116 & 143 & 142 & 139 & 102 \\
\hline 45 & 21 & 11 & 13 & 5 & $\mathbf{0}$ \\
\hline 58 & 15 & 19 & 8 & 5 & 2 \\
\hline 64 & 8 & 8 & 4 & 11 & $\mathbf{0}$ \\
\hline Total & 160 & 181 & 167 & 160 & 104 \\
\hline \multicolumn{6}{|c|}{ Deprivation } \\
\hline 31 & 0 & 0 & 0 & 0 & 0 \\
\hline 45 & 2 & 0 & 0 & 0 & $\mathbf{0}$ \\
\hline 51 & 2 & 0 & 1 & 0 & 0 \\
\hline 62 & 0 & 0 & 0 & $\mathbf{0}$ & $\mathbf{0}$ \\
\hline Total & 4 & 0 & 1 & 0 & $\mathbf{0}$ \\
\hline \multicolumn{6}{|c|}{ Deprivation } \\
\hline 32 & 0 & 0 & 0 & 6 & $\mathbf{0}$ \\
\hline 58 & 1 & 0 & 0 & 0 & $\mathbf{0}$ \\
\hline 64 & 6 & 0 & 0 & 1 & 1 \\
\hline 65 & 0 & 0 & 1 & $\mathbf{0}$ & $\mathbf{0}$ \\
\hline Total & 7 & 0 & 1 & 7 & 1 \\
\hline
\end{tabular}




\section{DISCUSSION}

Valenstein et al's (1969) report that ESLH animals continued to respond to a previously full, but now empty, drinking tube was essentially replicated. The reasons for this unusual behavior are open to further experimentation, since this experiment demonstrated that experience and deprivation play only a small role, if any, in influencing the deprived animal to lick on empty drinking tubes that have previously held liquid food. In addition, the data of this experiment show that the empty tube drinking behavior diminishes rapidly over a 5 -min period of stimulation. This effect seems to result from learning and not from an adaptation effect, since all animals switched to the eating of the wet mash and did not stop responding.

This experiment again emphasizes that there are basic differences between the consummatory behaviors produced by ESLH and deprivation. While this experiment does not suggest any solution as to the nature of ESLH-elicited behaviors, the persistence of the responding in the face of nonreinforcement is striking. The most parsimonious explanation of this result is that ESLH facilitates motor pathway responsiveness of well-established or dominant responses (Wayner, 1970; Valenstein et al, 1969). Theoretical explanations that attempt to explain ESLH-elicited behavior by relying on motivational concepts cannot easily explain this data.

\section{REFERENCES}

KRIEG, W. Accurate placement of minute lesions in the brain of the albino rat. Quarterly Bulletin of Northwestern Eniversity Medical School, 1946, 20 . 199-208.

Le MAGNEN, J. Habits and food intake. In Food and water intake (Vol. 1) of Alimentary canal, Section 6 of The handbook of physiology. Washington, D.C: American Physiological Society, 1967. Pp. 11-30.

MOLL, R. P. The effect of drive level on acquisition of the consummatory response. Journal of Comparative \& Physiological Psychology, 1959, 52, 116-119.

SIEGEL, S. Nonparametric statistics. New York: McGraw-Hill, 1956.

VALENSTEIN, E. S., COX, W. C., \& KAKOLEWSKI, J. W. In J. Tapp (Ed.) Reinforcement and behavior. New York: Academic Press, 1969.

WAYNER, M. J. Motor control functions of the lateral hypothalamus and adjunctive behavior. Physiology \& Behavior, 1970, 5, 1319-1325.
(Moskowitz, 1970). What remains is to examine animal preferences for these two compounds.

\section{SUBJECTS}

Naive male Holtzman albino rats, 130 days old at the beginning of testing, were housed individually, with standard (Big Red) laboratory chow available ad lib.

\section{APPARATUS}

Commercial grade mannitol and sorbitol (provided by the Nestlés Company) were used to prepare solutions with tap water on a weight-by-volume basis following the recommendations of Pfaffmann, Young, Dethier, Richter, \& Stellar (1954). Solutions were presented in inverted $100-\mathrm{ml}$ graduated cylinders fitted with glass drinking spouts. Solution concentrations of $7.5 \%$ were chosen to approximate those of glucose, which are known to be preferred over water and over a $0.25 \%$ saccharin solution.

\section{PROCEDURE}

Preference tests were $24 \mathrm{~h}$ in duration. Intake of each solution was recorded to the nearest $0.5 \mathrm{ml}$. If necessary, drinking tubes were refilled from refrigerated stock. When solutions were presented for more than one day, positions of the tubes were alternated to inhibit the development of position preferences.

Because of the small number of Ss, medians of percent intake were computed. Variability of the data was determined by computing a standard error of the median (Peatman, 1963).

Two groups of five rats each were given three-bottle choices of water, $7.5 \%$ mannitol, and $15 \%$ mannitol, or of water, $7.5 \%$ and $15 \%$ sorbitol for 3 days. On the fourth day, water was not available. On the fifth and sixth days, the two groups were given a two-bottle choice of $0.25 \%$ saccharin or $7.5 \%$ mannitol and saccharin or $7.5 \%$ sorbitol, respectively. The data from these two groups are referred to later in Fig. 1 as Parts A, B, D, F, and G.

Additional rats received choices as follows: Days 1-3, a three-bottle choice of water, mannitol, or sorbitol in each of three bottles; Day 4, mannitol or sorbitol alone; and Days 5 and 6, choices of saccharin and mannitol or saccharin and sorbitol. The data from these $S$ s are incorporated in Fig. 1, Parts C, E, F, and $\mathrm{G}$.

The experimental design is unfortunately complicated for several reasons. First, rats and chemical supplies were limited. Mannitol, for example, has been discontinued by Nestlés as a commercial sweetener and therefore is no longer available. Second, in a preference experiment of this type, where unexpected aversions
Industry has found that mannitol and sorbitol are acceptable as both mannitol and sorbitol to be about $80 \%$ as sweet as glucose-under conditions of equal concentrations 